\title{
Modulating microglia
}

\section{$c 6$}

\section{these findings indicate that microglia can regulate the severity of EAE via VEGFB or TGFa signals to astrocytes}

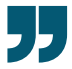

Under certain conditions, astrocytes express pro-inflammatory molecules and may have neurotoxic effects. Microglia regulate these astrocytic activities but the underlying mechanisms are incompletely understood. Rothhammer et al. now identify microglial-cell-derived regulators of astrocytes in mice with experimental autoimmune encephalitis (EAE), often used as a model of multiple sclerosis (MS), and show that, in this context, microglia may be regulated by microbial metabolites.

Previous studies have indicated that, in EAE, the pathogenic activities of astrocytes can be regulated by microbial metabolites of dietary tryptophan that are agonists of the aryl hydrocarbon receptor (AHR). Rothhammer et al. generated mice in which microglial Ahr could be conditionally knocked out to examine how AHR in these cells affects inflammation.

Following EAE induction, these mice showed greater disease severity than control animals, and this exacerbation of EAE was linked to increases in the recruitment of monocytes into the CNS and in demyelination. Moreover, microglialcell $A h r$ deletion in mice with EAE

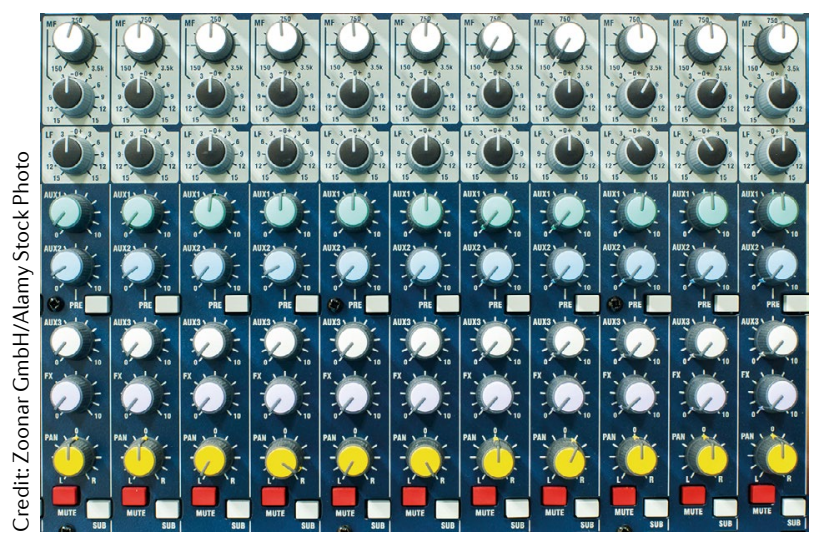

was associated with the upregulation of various genes involved in microglial activation, as well as increased expression of genes linked to neurodegeneration and inflammation in both microglia and astrocytes.

These data suggested that microglial AHR activation negatively regulates EAE, possibly through effects on astrocytes. The authors conducted a bioinformatics analysis revealing that microglial-cell-produced transforming growth factor- $\alpha$ (TGF $\alpha$ ) and vascular endothelial growth factor B (VEGFB) were candidate regulators of two transcription modules in astrocytes during EAE. Interestingly, deletion or inhibition of AHR in microglia increased Vegfb expression and decreased Tgfa expression in mice with EAE. Moreover, in cultured astrocytes, administration of VEGFB increased pro-inflammatory molecule expression, whereas application of TGFa reduced it. Last, pretreatment of astrocytes with VEGFB increased the toxicity of astrocyte-conditioned medium when it was applied to neurons and oligodendrocytes, and this effect could be inhibited by co-application of TGFa with VEGFB during the pretreatment. Thus, these data indicate that VEGFB and TGFa are key regulators of the pathogenic activities of astrocytes.

To complement the cell-culture work, the authors investigated the effect of knocking down microglial TGFa or VEGFB in mice with EAE through use of a virus-based method. In line with the other data, TGFa knockdown increased disease severity, whereas VEGFB knockdown improved the condition. Thus, these findings indicate that microglia can regulate the severity of EAE via VEGFB or TGFa signals to astrocytes.

The authors next examined how microglia are regulated. They found that control mice with EAE that were fed a tryptophan-depleted diet failed to show disease recovery, and this effect could be rescued by feeding these animals with tryptophan metabolites. This feeding strategy did not have any effect on mice with EAE that lacked microglial AHR and had a tryptophan-depleted diet. Indeed, EAE-induced mice in which $A h r$ was conditionally knocked out in microglia or astrocytes showed greater disease severity with tryptophan depletion. Together, these findings suggest that dietary tryptophan metabolites modulate inflammation in EAE via AHR in microglia and astrocytes.

Next, the authors conducted experiments in human samples. In primary cultures of human microglia, administration of a tryptophan metabolite activated AHR, leading to an increase in $T g f a$ expression, a decrease in Vegfb expression and suppression of the genes linked to inflammation and neurotoxicity. Moreover, in cultured human astrocytes, VEGFB and TGF $\alpha$ promoted and suppressed the expression of pro-inflammatory genes, respectively. Last, in brain tissue from people with MS, the ratio of VEGFB:TGFa expression was higher in chronic lesions than in normal-appearing white matter. Together, these data indicate that the mechanism uncovered in EAE may have relevance to human disease.

Overall, these data reveal a mechanism by which dietary metabolites may modulate the activities of glia to influence neuroinflammatory pathology.

Darran Yates

ORIGINAL ARTICLE Rothhammer, V. et al. Microglial control of astrocytes in response to microbial metabolites. Nature 557, 724-728 (2018) 